\title{
ATIVIDADES LARVICIDA E ANTICOLINESTERÁSICA DE PLANTAS DO GÊNERO Kalanchoe
}

\section{Maria Teresa Salles Trevisan*, Maria Zeneide Barbosa Bezerra, Gilvandete Maria Pinheiro Santiago e Chistiane Mendes Feitosa}

Departamento de Química Orgânica e Inorgânica, Universidade Federal do Ceará, CP 12200, 60021-970 Fortaleza - CE, Brasil Robert Verpoorte

Division of Pharmacognosy, Leiden/Amsterdam Center for Drug Research, Gorlaeus Laboratories, Leiden University, Leiden, The Netherlands

\section{Raimundo Braz Filho}

Setor de Química de Produtos Naturais, Centro de Ciência e Tecnologia, Universidade Estadual do Norte Fluminense, Av. Alberto Lamego, 2000, 28013-600 Campos - RJ, Brasil

Recebido em 25/8/04; aceito em 28/9/05; publicado na web em 14/3/06

LARVICIDES AND ACETYLCHOLINESTERASE INHIBITORS FROM Kalanchoe SPECIES. Acetylcholine esterase inhibitors are successfully used to treat the symptoms of Alzheimer's disease. Extracts of three Kalanchoe species (K. brasiliensis, K. pinnata and $K$. gastonis-bornieri) showed acetylcholine esterase inhibitory effects and a toxic effect on Aedes aegypti larvae. Here we describe the bioassay guided fractionation of extracts of the most active extracts (K. brasiliensis) which resulted in the isolation of an active mixture of three flavonoids: 8-methoxyquercetin, 3,7-di-O-rhamnopyranoside and 8-methoxykaempferol-3,7-di-Orhamnopyranoside. On TLC these flavonoids showed an acetylcholine esterase inhibitory effect.

Keywords: Kalanchoe species; flavonol glycosides; Alzheimer's disease.

\section{INTRODUÇÃO}

As espécies Kalanchoe brasiliensis Camb., K. pinnata Pers. e K. gastonis-bournieri Raym-Hamet et Perrier, pertencentes à família das crassuláceas, são popularmente conhecidas, respectivamente, por courama branca, courama vermelha e courama compri$\mathrm{da}^{1}$. O uso de espécies do gênero Kalanchoe para tratamento de artrites e úlceras gástricas é relatado principalmente na China e na África. O extrato das suas folhas revelou ações antifúngica, bactericida, analgésica e antiinflamatória ${ }^{2}$.

$\mathrm{O}$ extrato hidroalcoólico das folhas frescas da espécie $K$. brasiliensis Camb. demonstrou propriedade inibitória da colinesterase em experimentos realizados com reto abdominal isolado de sapos. Nestes experimentos, o efeito inibitório da $d$-tubocurarina (1) nas respostas contráteis provocadas por acetilcolina (2) foi efetivamente bloqueado pelo extrato. $\mathrm{O}$ mesmo efeito inibitório ocorre com a prostigmina (3), um anticolinesterásico conhecido. A observação destes efeitos possibilitou: atribuir ao extrato da planta uma atividade anticolinesterásica e, considerar que flavonóides glicosilados, revelados através de uma abordagem fitoquímica do extrato hidroalcoólico, poderiam ser os responsáveis por este efeito inibitório da colinesterase ${ }^{3}$.

Flavonóides glicosilados contendo a 3,3',4',5,7-pentaidroxi-6metoxiflavona (patuletina) como aglicona foram isolados da espécie K. brasiliensis, coletada durante a primavera de 1992 no Rio de Janeiro-RJ ${ }^{4}$, cidade que pertence à região sudeste do País. Essa região está submetida a condições edafo-climáticas significativamente diferentes das observadas em Fortaleza-CE, onde foi coletado o espécime utilizado no nosso estudo.

Resultados preliminares justificaram investigações adicionais com a referida planta K.brasiliensis e a seleção de mais outras duas espécies do mesmo gênero - K. pinnata Pers. e K. gastonis-

*e-mail: trevisan@ufc.br bournieri Raym-Hamet et Perrier - para verificação, in vitro, da atividade inibitória de enzima acetilcolinesterase (AChE).

O mal de Alzheimer é uma doença neurodegenerativa que atinge primeiramente a memória e, posteriormente, a capacidade de raciocínio e a comunicação. O quadro de sinais e sintomas dessa doença está associado à redução de neurotransmissores cerebrais, como acetilcolina (2), noradrenalina (4) e serotonina (5) ${ }^{5}$. O tratamento para o mal de Alzheimer é sintomático e consiste justamente na tentativa de restauração da função colinérgica. Dessa forma, a elevação do nível da acetilcolina pode se mostrar útil para amenizar a deficiência da aprendizagem, um dos sinais da doença ${ }^{6}$.

Inibidores da acetilcolinesterase são amplamente usados no tratamento da doença, procedimento que se baseia na hipótese colinérgica. A galantamina (6), um alcalóide isolado de plantas da família Amaryllidaceae, apresenta uma longa ação seletiva, rever-

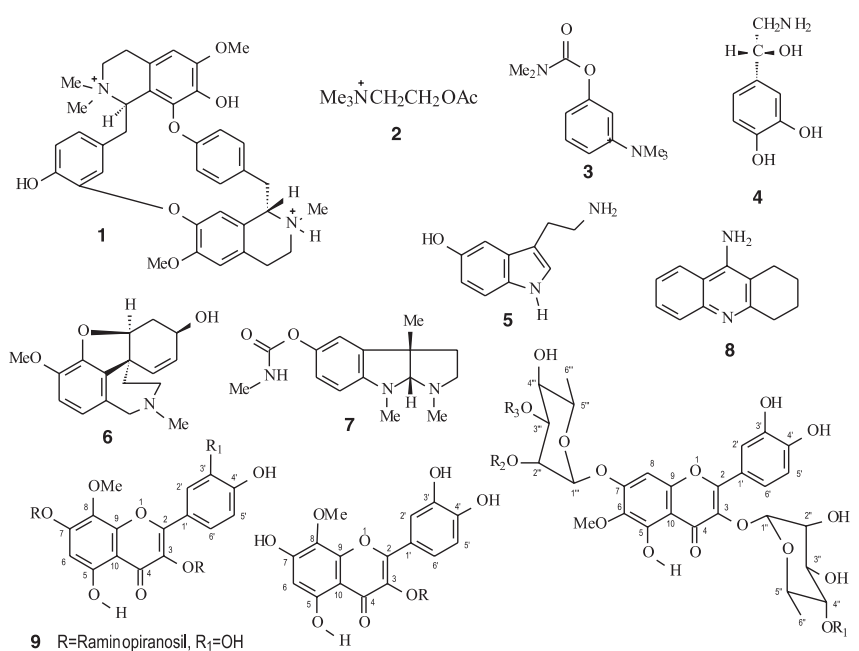

$10 \mathrm{R}=$ Raminopiranosil, $\mathrm{R}_{1}=\mathrm{H}$
$12 \mathrm{R}_{1}=\mathrm{R}_{2}=\mathrm{R}_{3}=\mathrm{H}$ 
sível e competitiva para inibir a acetilcolinesterase. A galantamina (6) é considerada mais efetiva no tratamento da doença de Alzheimer que a fisostigmina (7) e a tacrina $(\mathbf{8})^{7,8}$, que são as substâncias atualmente utilizadas. Esses medicamentos estão disponíveis no mercado a um preço relativamente oneroso, o que torna a busca de novos inibidores de origem vegetal uma alternativa interessante, na medida em que estes seriam economicamente mais viáveis.

Atualmente, há uma atividade de pesquisa relativamente intensa na busca de novos inibidores em extratos de plantas ${ }^{8,9}$, com especial interesse no isolamento e na identificação de novos inibidores da AChE.

O presente trabalho descreve os resultados experimentais das atividades anticolinesterásica e larvicida de extratos de três espécies do gênero Kalanchoe e de flavonóides glicosilados isolados do extrato acetato de etila (AcOEt) de K. brasiliensis.

\section{RESULTADOS E DISCUSSÃO}

\section{Ensaio em CCD/AChE}

A constatação da inibição da AChE é possível seguindo-se a metodologia de Elmann ${ }^{10}$, adaptada por Rhee et al. ${ }^{8}$, para cromatografia em camada delgada fina. Neste ensaio, utiliza-se a solução dos reagentes ácido 5,5'-ditiobis-2-nitrobenzóico (DTNB) e iodeto de acetilcolina (ATCI) em tampão e, posteriormente, aplica-se a enzima $\mathrm{AChE}(3 \mathrm{U} / \mathrm{mL})$. A visualização da inibição dá-se pela observação de halos brancos. A mistura dos flavonóides isolados do extrato AcOEt de $K$. brasiliensis foi bio-guiada pela inibição da enzima. A presença dos flavonóides ativos é confirmada pelo aparecimento de halo branco ${ }^{8}$.

\section{Inibição da acetilcolinesterase em ensaio de microplaca}

O ensaio enzimático em CCD é um teste qualitativo. Para obtenção de resultados quantitativos, é necessária a utilização dos ensaios em microplaca (absorbância a $405 \mathrm{~nm}$ ). Das amostras testadas, os extratos AcOEt e metanólico $(\mathrm{MeOH})$ de $K$. brasiliensis e o extrato AcOEt de K. pinnata apresentaram inibição de $100 \%$ no ensaio de microplaca na concentração de $2 \mathrm{mg} / \mathrm{mL}$. A inibição apresentada pelo extrato de AcOEt de K. gastonis-bournieri RaymHamet et Perrier, na mesma concentração, 82\%, indica esse extrato como promissor para futuros fracionamentos (Tabela 1).

$\mathrm{O}$ extrato AcOEt de K. brasiliensis foi então selecionado para fracionamento bio-guiado, por apresentar inibição enzimática de $100 \%$ em microplaca, nos extratos AcOEt e MeOH. Estudos realizados com reto abdominal isolado de sapos também demonstraram a propriedade inibitória da colinesterase pelo extrato hidroalcóolico ${ }^{3}$.

$\mathrm{O}$ extrato EtOAc de $K$. brasiliensis tem valor de $\mathrm{CI}_{50}=0,16$ $\mathrm{mg}$, o potente inibidor da $\mathrm{AChE}$, a galantamina, que já é utilizada como medicamento, apresenta o valor de $\mathrm{CI}_{50}=0,37 \times 10^{-3} \mathrm{mg}$.
Tabela 1. Resultados de microplaca (inibição) para extratos AcOEt e $\mathrm{MeOH}$ de espécies de Kalanchoe

\begin{tabular}{|c|c|c|}
\hline \multirow[b]{2}{*}{ Espécie (Exsicata) } & \multicolumn{2}{|c|}{ Microplaca (\%) $2 \mathrm{mg} / \mathrm{mL}$} \\
\hline & AcOEt & $\mathrm{MeOH}$ \\
\hline Kalanchoe brasiliensis Camb.(14657) & 100 & 100 \\
\hline Kalanchoe pinnata Pers. (31144) & 100 & 15 \\
\hline Kalanchoe gastonis-bournieri & 82 & 2 \\
\hline
\end{tabular}

Raym-Hamet et Perrier (13782)

Ensaios para $25 \mu \mathrm{L}$, testados com 1 e $5 \mathrm{mg}$ em $1 \mathrm{~mL}$ de metanol.

Observe-se que o resultado é bastante significativo por se tratar de um extrato bruto.

\section{Ensaios de atividade larvicida}

Os ensaios de atividade larvicida foram realizados utilizandose a larva do mosquito Aedes aegypti. Os extratos das folhas de três espécies e dos flavonóides obtidos através do isolamento bio-guiado do extrato AcOEt de $K$. brasiliensis foram submetidos à investigação de atividade biológica em três concentrações diferentes, na ordem decrescente de 500, 250 e 100 ppm (Tabela 2).

Os extratos $n$-hexânicos das folhas de $K$. brasiliensis e $K$. pinnata revelaram, respectivamente, os valores de $\mathrm{DL}_{50} 269,15$ e 301,99 ppm; os extratos AcOEt revelaram valores de $\mathrm{DL}_{50} 281,83$ ppm para $K$. pinnata; na concentração de 250 ppm, foi letal $\left(\mathrm{DL}_{50}<\right.$ $250 \mathrm{ppm})$ para $K$. brasiliensis. Esses resultados foram considerados significativos por se tratar de extratos brutos. Os flavonóides isolados de $K$. brasiliensis foram testados na concentração de 50 ppm e não apresentaram efeito larvicida.

\section{Determinação estrutural dos flavonóides isolados do extrato AcOEt de $K$. brasiliensis}

Os flavonóides 3,7-di- $O$ - $\alpha$-L-raminopiranosil-8-metoxiquercetina (9) e 3,7-di-O- $\alpha$-L-raminopiranosil 8-metoxikanferol (10), isolados como uma mistura através de fracionamento bio-guiado, foram caracterizados principalmente com base nos espectros de RMN ${ }^{1} \mathrm{H}$ e ${ }^{13} \mathrm{C}$ (Tabela 3), envolvendo comparação com valores descritos na literatura para o flavonóide 12, isolado de K.brasiliensis ${ }^{4}$.

$\mathrm{O}$ espectro de $\mathrm{RMN}^{1} \mathrm{H}$ mostrou os sinais dos átomos de hidrogênio do anel B de 9 em $\delta_{\mathrm{H}} 7,43$ (d, $J=2,0, \mathrm{H}^{2}$ ') $) 6,97$ (d, $J=8,4$, H-5') e 7,39 (dd, $J=8,4$ e 2,0, H-6'), e de $\mathbf{1 0}$ em $\delta_{\mathrm{H}}$ 7,82 (d, $J=8,8$, H-2'/H-6') e 6,98 (d, $J=8,8$, H-3'/H-5'). A atribuição inequívoca dos deslocamentos químicos dos átomos de hidrogênio e carbono de 9 e 10 baseou-se também em interações heteronucleares a longa distância de carbono e hidrogênio $\left({ }^{2} J_{\mathrm{CH}}\right.$ e $\left.{ }^{3} J_{\mathrm{CH}}\right)$, reveladas pelo espectro 2D HMBC (Tabela 3).

Com base nas intensidades relativas reveladas pela integração computacional dos sinais dos átomos de hidrogênio H-5' $\left(\delta_{\mathrm{H}} 6,97\right)$

Tabela 2. Porcentagem de mortes, em testes de atividade contra a larva do mosquito Aedes aegypti de folhas das espécies de Kalanchoe, com indicação dos extratos e das concentrações utilizadas

\begin{tabular}{|c|c|c|c|c|c|c|}
\hline \multirow[b]{3}{*}{ Espécie } & \multicolumn{6}{|c|}{ Extrato } \\
\hline & \multicolumn{2}{|c|}{ Hexânico (\%) } & \multicolumn{3}{|c|}{ AcOEt $(\%)$} & \multirow{2}{*}{$\frac{\mathrm{MeOH}(\%)}{500 \mathrm{ppm}}$} \\
\hline & $500 \mathrm{ppm}$ & $250 \mathrm{ppm}$ & $500 \mathrm{ppm}$ & $250 \mathrm{ppm}$ & $100 \mathrm{ppm}$ & \\
\hline Kalanchoe brasiliensis Camb. & 100 & 25 & 100 & 100 & 0 & 40 \\
\hline Kalanchoe pinnata Pers. & 100 & 3 & 100 & 13 & - & 0 \\
\hline $\begin{array}{l}\text { Kalanchoe gastonis-bournieri } \\
\text { Raym-Hamet et Perrier }\end{array}$ & 100 & 0 & 100 & 0 & - & 44 \\
\hline
\end{tabular}


Tabela 3. Dados de $\mathrm{RMN}{ }^{13} \mathrm{C}$ e $\mathrm{RMN}{ }^{1} \mathrm{H}$ dos flavonóides 9 e $\mathbf{1 0}$ e RMN ${ }^{1} \mathrm{H}$ de $\mathbf{1 1}$ comparados com os valores de 12, usando $\mathrm{CD}_{3} \mathrm{OD}$ como solvente. Deslocamentos químicos em $\delta_{\mathrm{C}}$ e $\delta_{\mathrm{H}}(\mathrm{ppm})$ e constantes de acoplamento ( $J$, entre parênteses) em Hz*

\begin{tabular}{|c|c|c|c|c|c|c|}
\hline & & 9 & & & 11 & 12 \\
\hline & $\delta_{\mathrm{C}}$ & $\delta_{\mathrm{H}}$ & $\delta_{\mathrm{C}}$ & $\delta_{\mathrm{H}}$ & $\delta_{\mathrm{H}}$ & $\delta_{\mathrm{C}}$ \\
\hline $\mathbf{C}$ & & & & & & \\
\hline 2 & 160,00 & - & & & & 159,6 \\
\hline 3 & 136,40 & - & & & & 136,3 \\
\hline 4 & 179,99 & - & & & & 179,8 \\
\hline 5 & 157,69 & - & & & & 150,0 \\
\hline 6 & 100,03 & $6,67(s)$ & 100,05 & $6,67(s)$ & $6,65(\mathrm{~s})$ & 130,8 \\
\hline 7 & 156,35 & & & & & 156,2 \\
\hline 8 & 130,98 & - & & & & 99,7 \\
\hline 9 & 150,11 & - & & & & 157,6 \\
\hline 10 & 107,38 & - & & & & 107,2 \\
\hline $1^{\prime}$ & 122,80 & - & & & & 122,8 \\
\hline 2 ' & 117,21 & $7,43(\mathrm{~d}, 2,0)$ & 132,15 & $7,82(\mathrm{~d}, 8,8)$ & $7,83(\mathrm{~d}, 2,0)$ & 117,0 \\
\hline $3^{\prime}$ & 146,44 & - & 116,84 & $6,98(\mathrm{~d}, 8,8)$ & & 146,3 \\
\hline $4^{\prime}$ & 150,11 & - & & & & 149,9 \\
\hline 5 , & 116,74 & $6,97(\mathrm{~d}, 8,4)$ & 116,84 & $6,98(\mathrm{~d}, 8,8)$ & $6,91(\mathrm{~d}, 8,6)$ & 116,4 \\
\hline 6 ' & 123,31 & $7,39(\mathrm{dd}, 8,4 ; 2,0)$ & 132,15 & $7,82(\mathrm{~d}, 8,8)$ & $7,75(\mathrm{dd}, 8,6,2,0)$ & 123,2 \\
\hline $1 "$ & 103,62 & $5,34(\mathrm{~s})$ & & & $5,57(\mathrm{~s})$ & 103,6 \\
\hline $2 "$ & 71,88 & $4,23(\mathrm{~s})$ & & & $4,10(\mathrm{~s})$ & 71,9 \\
\hline $3 "$ & 72,11 & $3,79(\mathrm{dd}, 3,2 ; 9,3)$ & & & $3,91(\mathrm{dd}, 3,3,9,3)$ & 72,0 \\
\hline $4 "$ & 73,22 & $3,36(t, 9,3)$ & & & $3,52(\mathrm{t}, 9,3)$ & 73,2 \\
\hline $5 "$ & 72,20 & $3,43(\mathrm{qd}, 9,3 ; 6,0)$ & & & $3,69(\mathrm{qd}, 9,3,6,0)$ & 72,0 \\
\hline $6 "$ & 17,69 & $0,96(\mathrm{~d}, 6,0)$ & & & $1,27(\mathrm{~d}, 6,0)$ & 17,6 \\
\hline $1, "$ & 100,43 & $5,67(\mathrm{~s})$ & & & & 100,3 \\
\hline $2 " ”$ & 71,74 & $4,14(\mathrm{~s})$ & & & & 71,7 \\
\hline $3^{\prime \prime \prime}$ & 72,24 & $3,93(\mathrm{dd}, 3,3 ; 9,6)$ & & & & 72,1 \\
\hline $4{ }^{\prime \prime}$ & 73,56 & $3,53(\mathrm{t}, 9,6)$ & & & & 73,5 \\
\hline $5^{\prime \prime}$ & 71,52 & $3,67(q d, 9,6,6,2)$ & & & & 71,4 \\
\hline $6 "$, & 18,12 & $1,26(\mathrm{~d}, 6,2)$ & & & & 18,1 \\
\hline $\mathrm{CH}_{3}-6$ & - & & & & & 62,3 \\
\hline $\mathrm{CH}_{3}-8$ & 62,64 & $3,98(\mathrm{~s})$ & & & $3,94(\mathrm{~s})$ & - \\
\hline
\end{tabular}

* O número de átomos de hidrogênio ligado a cada carbono foi deduzido com base na análise comparativa dos espectros de $\mathrm{RMN}{ }^{13} \mathrm{C}\left\{{ }^{1} \mathrm{H}\right\}$ e RMN ${ }^{13} \mathrm{C}$-DEPT. Espectros 2D homonuclear ${ }^{1} \mathrm{H}-{ }^{1} \mathrm{H}-\mathrm{COSY}$ e heteronuclear HMQC e HMBC foram também utilizados na atribuição dos deslocamentos químicos de $\mathbf{9}$ e $\mathbf{1 0}$. Os valores dos deslocamentos químicos e das constantes de acoplamento $(J)$ dos átomos de hidrogênio foram obtidos do espectro $1 \mathrm{D}$ de $\mathrm{RMN}{ }^{1} \mathrm{H}$; s=singleto; t=tripleto; d=dubleto; dd=duplo dubleto; qd=quadrupleto; $J$ em Hertz.

de 9 e H-2'/H-6' $\left(\delta_{\mathrm{H}} 7,82\right)$ de $\mathbf{1 0}$ no espectro $1 \mathrm{D}$ de $\mathrm{RMN}{ }^{1} \mathrm{H}$ da mistura calcularam-se as percentagens aproximadas dos dois flavonóis: $90 \%$ (9) e 10\% (10).

A comparação dos deslocamentos químicos dos átomos de carbono de 9 e $\mathbf{1 2}^{4}$ (Tabela 3) revela valores praticamente idênticos. Verificam-se atribuições discordantes para os átomos de carbono C-5 [ $\delta_{\mathrm{C}} 157,69(\mathbf{9})$ e $\left.150,0(\mathbf{1 2})\right]$ e C-9 [ $\delta_{\mathrm{C}} 150,11(\mathbf{9})$ e $\left.157,6(\mathbf{1 2})\right]$, o que se justifica pelo efeito mesomérico decorrente da presença em posição orto do grupo metoxílico em C-8 (9, C-5 livre do efeito mesomérico de proteção do metoxílico em posição orto, $\left.\delta_{C} 157,69\right)$ e C-6 (12, C-5 protegido mesomericamente pela presença do metoxílico em posição orto, $\left.\delta_{\mathrm{C}} 150,0\right)$ na hipótese das duas serem realmente diferentes. A localização definitiva do grupo metoxílico no átomo de carbono C-8 de 9 baseou-se no espectro 2D HMBC, que mostrou acoplamento heteronuclear do carbono $C-5\left(\delta_{C} 157,69\right)$ com o hidrogênio H-6 $\left(\delta_{\mathrm{H}} 6,67,{ }^{2} J_{\mathrm{CH}}\right)$, e no deslocamento químico $\delta_{\mathrm{C}} 100,03$ atribuído ao carbono metínico $\mathrm{CH}-6$, que é significativamente diferente do deslocamento químico do $\mathrm{CH}-8$ de flavonóis sustentando três substituintes oxigenados no anel $\mathrm{A}^{4,11-14}$. Os dados obtidos dos espectros 1D e 2D de RMN permitiram também a atribuição inequívoca dos deslocamentos químicos dos átomos de hidrogênio e carbono de $\mathbf{9}$ e $\mathbf{1 0}$ (Tabela 3) e, obviamente, não afas- tam a possibilidade de $\mathbf{9}$ e $\mathbf{1 2}$ serem idênticas e sugerem reavaliação da estrutura proposta anteriormente ${ }^{4}$.

O flavonóide 3-O- $\alpha$-L-raminopiranosil-3,3',4',5,7-pentaidroxi8-metoxiflavona (11), isolado do extrato AcOEt de $K$. brasiliensis, não inibiu a AChE e a estrutura proposta (11) foi especulativamente proposta baseada somente no espectro de $\mathrm{RMN}{ }^{1} \mathrm{H}$ (Tabela 3), que se apresenta também em consonância biogenética com os flavonóides glicosilados $\mathbf{9}$ e $\mathbf{1 0}$ isolados da mesma espécie vegetal.

\section{CONCLUSÕES}

Das três espécies testadas, a $K$. brasiliensis apresentou os resultados mais satisfatórios, o que justificou a seleção do extrato AcOEt para o fracionamento bio-guiado. O estudo do extrato AcOEt, que inibiu a enzima em $100 \%$, apresentando resultado positivo em $\mathrm{CCD}$ e potencial larvicida com $\mathrm{DL}_{50}<250 \mathrm{ppm}$, levou ao isolamento da mistura dos flavonóides ativos: 3,7-di- $O$ - $\alpha$-Lraminopiranosíl 8-metoxiquercetina $(9)$ e 3,7-di- $O$-raminosil 8metoxikanferol (10). Confirmou-se, assim, a suposição de Fonteles de que a presença de flavonóides glicosídeos, detectada na abordagem fitoquímica do extrato hidroalcoólico de $K$. brasiliensis, poderia ser a responsável por esse efeito inibitório da colinesterase ${ }^{3}$. 
Apesar de inibir a enzima acetilcolinesterase, a mistura destes flavonóides não apresentou atividade larvicida na concentração de $50 \mathrm{ppm}$.

\section{PARTE EXPERIMENTAL}

\section{Plantas}

As folhas das espécies $K$. brasiliensis, K. pinnata e K. gastonis foram coletadas no Horto de Plantas Medicinais Francisco José de Abreu Matos, pertencente à Universidade Federal do Ceará (UFC), Fortaleza-CE, e as exsicatas encontram-se depositadas no Herbarium Prisco Bezerra, do Departamento de Biologia - UFC.

\section{Procedimento para obtenção dos extratos}

O material vegetal foi seco em estufa a $50{ }^{\circ} \mathrm{C}$ (quantidade inici$\mathrm{al}=1,43 \mathrm{~kg}$; quantidade final $=110 \mathrm{~g}$ ) e, posteriormente, submetido à extração a frio com os solventes n-hexano, acetato de etila e metanol, sucessivamente. Os solventes foram evaporados sob pressão reduzida, obtendo-se então os extratos hexânico, acetato de etila e metanólico das folhas.

\section{Isolamento dos flavonóides de Kalanchoe brasiliensis}

As folhas secas $(110 \mathrm{~g})$ de $K$. brasiliensis foram submetidas à extração a frio com os solventes n-hexano, AcOEt e metanol, resultando três extratos distintos. Posteriormente, estes extratos foram testados para avaliar a inibição da AChE em CCD. O teste com o extrato AcOEt, positivo para a referida inibição, foi então submetido à partição com os solventes n-hexano, diclorometano e AcOEt. Da fração AcOEt, que apresentou atividade em AChE positiva, obtida da partição, foram realizadas sucessivas colunas cromatográficas usando-se gel de sílica como adsorvente e solventes convencionais, como n-hexano, diclorometano, AcOEt e metanol, em forma pura ou mistura binária. A fração n-hexano-diclorometano (2:8) e n-hexano-diclorometano (8:2) forneceu $3 \mathrm{mg}$ do flavonóide 11 e 20 mg de uma mistura dos dois flavonóides 9 e 10. Esta mistura apresentou resultado positivo para inibição de AChE.

\section{Medida da atividade de acetilcolinesterase}

\section{Soluções padrões}

As seguintes soluções foram preparadas: (1) $50 \mathrm{mM}$ Tris/HCl pH 8; (2) $50 \mathrm{mM}$ Tris/ $\mathrm{HCl} \mathrm{pH} 8$, contendo $0,1 \%$ de albumina sérica bovina (BSA) fração V; (3) $50 \mathrm{mM}$ Tris/ $\mathrm{HCl} \mathrm{pH} 8$, contendo $0,1 \mathrm{M}$ de $\mathrm{NaCl}$ e $0,02 \mathrm{M}$ de $\mathrm{MgCl}_{2} \cdot 6 \mathrm{H}_{2} \mathrm{O}$; (4) $3 \mathrm{mM}$ de ácido 5,5'-ditiobis[2-nitrobenzóico] (DTNB ou reagente de Ellman), (5) $15 \mathrm{mM}$ de iodeto de acetilcolina (ACTI), (6) $1 \mathrm{mM}$ de ácido 5,5'-ditiobis-[2nitrobenzóico] (DTNB ou reagente de Ellman) e (7) $1 \mathrm{mM}$ de iodeto de acetiltiocolina (ACTI).

A enzima AChE liofilizada foi dissolvida na solução tampão (1) para fazer uma solução estoque $1000 \mathrm{U} / \mathrm{mL}$. Deixou-se a enzima na solução por $20 \mathrm{~min}$ e, posteriormente, para obtenção de uma solução homogênea, procedeu-se à agitação por mais um período de 10-15 min. Para as diluições posteriores, utilizou-se a solução tampão (2).

\section{Ensaio na microplaca}

Para a reação enzimática no ensaio de microplaca, utilizou-se uma leitora de microplaca Bio-Rad, modelo 3550 UV, absorbância a $405 \mathrm{~nm}$, baseado no método de Ellman ${ }^{10}$. A enzima hidrolisou o substrato acetiltiocolina, gerando como produto a tiocolina, que reage com o reagente de Ellman, produzindo 2-nitrobenzoato-5mercaptotiocolina e 5-tio-2-nitrobenzoato, que podem ser detectados a $405 \mathrm{~nm}$. Nas 96 cavidades da placa, adicionaram-se $25 \mu \mathrm{L}$ de (5); $125 \mu \mathrm{L}$ de (4); $50 \mu \mathrm{L}$ de (2); $25 \mu \mathrm{L}$ da amostra a ser analisada, dissolvida em $\mathrm{MeOH}$, diluída 10 vezes em (1); mediu-se a absorbância a cada 13 s por 5 vezes. Adicionaram-se $25 \mu \mathrm{L}$ da enzima $(0,22 \mathrm{U} / \mathrm{mL})$; mediu-se novamente a absorbância a cada $13 \mathrm{~s}$ por 8 vezes. Calculou-se a velocidade das reações utilizando-se o software Microplate Manager version 4.0 (Bio-Rad Laboratories). Corrigiram-se os aumentos em absorbância, devido à hidrólise espontânea, por meio da subtração da velocidade da reação antes de se adicionar a enzima da velocidade da reação depois de se adicionar a enzima. A porcentagem de inibição foi calculada pela comparação das velocidades das amostras em relação ao branco $(10 \% \mathrm{MeOH}$ no tampão 1).

\section{Ensaio em cromatografia de camada delgada $(C C D)$}

As amostras $(1,5-2,5 \mu \mathrm{L})$ foram aplicadas em CCD, DCAlufolien, Silicagel 60 F254, 0,2 mm Merck. Borrifou-se a placa com as soluções $(6)+(7)$, deixando por $3 \mathrm{~min}$. Após secar, borrifou-se a enzima $3 \mathrm{U} / \mathrm{mL}$ e, em $10 \mathrm{~min}$, apareceu a coloração amarela. Onde houve inibição da enzima, observou-se um halo bran$\mathrm{co}^{7}$. Em cerca de 20 a $30 \mathrm{~min}$, a coloração desapareceu.

\section{Ensaios de atividade larvicida contra a larva do mosquito Aedes} aegypti

Os testes com os extratos hexânico, acetato de etila e metanólico foram realizados nas concentrações de 500, 250 e 100 ppm (Tabela 2). As amostras foram dissolvidas em $0,3 \mathrm{~mL}$ de dimetilsulfóxido, completando-se o volume com água e larvas do mosquito no $3^{\circ}$. estágio, até a obtenção de $20 \mathrm{~mL}$. Após 24 h, as larvas mortas foram contadas. Realizaram-se os ensaios em duplicatas e em diferentes concentrações para posterior cálculo de $\mathrm{DL}_{50}$ (concentração da amostra capaz de matar $50 \%$ da população de larvas). O dimetilsulfóxido foi utilizado como controle na mesma concentração utilizada para dissolução da amostra.

\section{AGRADECIMENTOS}

Ao Prof. Dr. F. J. de A. Matos, pelo fornecimento das plantas, e aos órgãos financiadores CNPq, CAPES, FUNCAP e FAPERJ, pelas bolsas e auxílios concedidos.

\section{REFERÊNCIAS}

1. Matos, F. J. A.; Plantas da medicina popular do Nordeste; propriedades atribuídas e confirmadas, Edições UFC: Fortaleza, 1999.

2. Bergmann, B. R.; Costa, S. S; Moraes, V. L. G.; Nat. Prod. Res. in Brazil 1997, 49, 395

3. Fonteles, M. C.; Capelo, R. L.; Rao, V. S. N.; VII Simpósio de Plantas Medicinais, Brasil, 1982.

4. Bodo, J. B.; Costa, S. S.; Souza, M. L. M.; Moraes, V. L. G.; J. Nat. Prod. 1994, 57, 1503 .

5. Bryne, G. J. A.; Aust. J. Hosp. Pharm. 1998, 28, 261.

6. Perry, E. K.; British Medicinal Bulletin 1986, 42, 63

7. Ingkaninan, K.; de Best, C. M.; van der Heidjen, R.; Hofte, A. J. P.; Karabatak, B.; Irth, H.; Tjaden, U. R.; van der Greef, J.; Verpoorte, R.; J. Chromatogr., A 2000, 872, 61

8. Rhee, I. K.; Meent, M. V.; Ingkaninan, K.; Verpoorte, R.; J. Chromatogr., A 2001, 915, 217

9. Trevisan, M. T. S.; Macedo, F. V. V.; Meent, M. V.; Rhee, I. K.; Verpoorte, R.; Ouim. Nova 2003, 26, 301.

10. Ellman, G. L.; Biochem. Pharmacol. 1961, 7, 88.

11. Wu, T. S.; Furukawa, H.; Phytochemistry 1983, 22, 1061.

12. Roitman, J. N.; James, L. F.; Phytochemistry 1985, 24, 835.

13. Merfort, I.; Wendisch, D.; Planta Med. 1987, 5, 434.

14. Aritomi, M.; Komori, T.; Kawasaki, T.; Phytochemistry 1986, 25, 231. 